ISSN 1794-9831

E-ISSN 2322-7028

Vol. 12 No. 2

Jul - Dic 2015

Cúcuta, Colombia

Recibido:

25 de Mayo

de 2015

Aceptado por

pares:

11 de Noviembre de 2015

Aprobado:

1 de Diciembre de 2015
* Bacterióloga. Magister en Salud Ocupacional. Candidata a Magister en Epidemiología. Docente Ocasional. Universidad de los Llanos. Villavicencio, Colombia. Correo electrónico: lplata@unillanos. edu.co

** Descriptores en Ciencias de la Salud (DeCS), en la página http:// decs.bvs.br/E/ homepagee.htm de la Biblioteca virtual en salud del proyecto BIREME, de la Organización Mundial de la Salud y de la Organización Panamericana de Salud.

\title{
Factores asociados a la no adherencia al tratamiento anti tuberculosis
}

\author{
Laura Inés Plata-Casas*
}

\section{RESÚMEN}

Objetivo: describir los factores de riesgo asociados a la no adherencia al tratamiento en los pacientes adscritos al programa de tuberculosis en el Municipio de Villavicencio, cohorte 2012. Materiales y Métodos: se ha realizado un estudio descriptivo de corte transversal con enfoque cuantitativo. Se obtuvieron datos de caracterización sociodemográfica sobre factores de riesgo de personas que abandonaron el tratamiento para la enfermedad- cohorte 2012- mediante la aplicación de un instrumento diseñado por el investigador y revisado por expertos. Los datos fueron tabulados y analizados mediante la herramienta Microsoft Excel ${ }^{\circledR} 2007$ y Epi-info 7.0. Resultados: el 75\% de los pacientes pertenecen al género masculino; el promedio de edad es de 30 años, el 63\% pertenecen al régimen subsidiado de salud. Las principales causas de abandono fueron: efectos adversos a los medicamentos (58\%), inadecuada atención del personal de salud (43\%), dificultad de acceso al servicio (32\%). Los factores sociales encontrados fueron hacinamiento $67 \%$ y desempleo $62 \%$. Conclusiones: algunos factores no modificables como la edad y el género, así como factores modificables como estilos de vida inadecuados y efectos adversos; cuyo control mejoraría el cumplimiento; contribuyen al abandono del tratamiento. Las variables psicológicas y sociales deben ser evaluadas para que se conviertan en predictores de probables abandonos o irregularidades en el tratamiento antituberculoso.

PALABRAS CLAVE: mycobacterium tuberculosis, negativa del paciente al tratamiento, tuberculosis ${ }^{* *}$.

\footnotetext{
Para citar este artículo / To reference this article / Para citar este artigo

Plata-Casas LI. Factores asociados a la no adherencia al tratamiento anti tuberculosis. Rev. cienc. cuidad. 2015; 12(2): $26-38$.
} 


\section{Factors associated with non-adherence to anti- tuberculosis treatment}

\section{ABSTRACT}

Goal: to describe the risk factors associated with non-adherence to the treatment in patients enrolled in the tuberculosis program in Villavicencio city, cohort 2012. Materials and Methods: a descriptive cross-sectional study with quantitative approach. Socio-demographic data and characterization of risk factors for people who discontinued treatment for the disease. Datawere obtained by applying 2012 cohort instrument designed by the researcher and reviewed by experts. Data were tabulated and analyzed using Microsoft Excel 2007 and Epi-info 7.0 tool. Results: $75 \%$ of patients are male; the average age is 30 years, $63 \%$ of them belong to the subsidized health system. The main causes of abandonment were: adverse effects to medicines (58\%), an inadequate health staff care (43\%), some difficulties to access to the service (32\%). The social factors were overcrowding found $67 \%$ and $62 \%$ unemployment. Conclusions: non-modifiable factors such as age and gender, as well as modifiable factors such as inadequate lifestyle and adverse effects; People in charge of the control would improve compliance; contribute to cessation of therapy. The psychological and social variables should be evaluated to become predictors of probable dropouts or irregularities in TB treatment.

KEY WORDS: mycobacterium tuberculosis, the patient refused treatment, tuberculosis. 
ISSN 1794-9831

E-ISSN 2322-7028

Vol. 12 No. 2

Jul - Dic 2015

Cúcuta, Colombia

\section{Fatores associados à não-adesão ao tratamento anti-tuberculose}

\section{RESUMO}

Objetivo: descrever os fatores de risco associados à não-adesão ao tratamento em participar do programa de tuberculose na cidade de Villavicencio paciente coorte de 2012. Materiais e Métodos: um estudo descritivo transversal, com abordagem quantitativa. dados sóciodemográficos e caracterização dos fatores de risco para as pessoas que interromperam o tratamento para a doença foram obtidos através da aplicação de coorte 2012 instrumento concebido pelo investigador e revisados por especialistas. Os dados foram tabulados e analisados usando o Microsoft Excel 2007 e Epi-info 7.0 ferramenta. Resultados: 75\% dos pacientes são masculinos; a média de idade é de 30 anos, $63 \%$ pertencem ao sistema de saúde subsidiado. As principais causas de abandono foram: efeitos adversos a medicamentos (58\%), profissionais de saúde inadequada (43\%), dificuldade de acesso ao serviço (32\%). Os fatores sociais foram encontrados superlotação $67 \% \mathrm{e} \%$ de desemprego 62 . Conclusões: os fatores não modificáveis, como idade e sexo, bem como fatores modificáveis, tais como estilos de vida inadequados e efeitos adversos; cujo controle seria melhorar o cumprimento; contribuir para a interrupção da terapia. variáveis psicológicas e sociais devem ser avaliados para se tornar preditores de abandono prováveis ou irregularidades no tratamento da TB.

PALAVRAS-CHAVE: mycobacterium tuberculosis, o paciente recusou o tratamento, a tuberculose. 


\section{INTRODUCCIÓN}

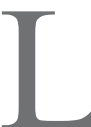

a tuberculosis (TB) se incluye entre las enfermedades infecciosas más comunes en el mundo, es la segunda causa de muerte por un único agente infeccioso en personas que padecen el virus de la inmunodeficiencia humana (VIH) (1). Es una enfermedad infecciosa crónica causada por el complejo Mycobacterium tuberculosis (MT), el cual puede afectar cualquier órgano o tejido; sin embargo, la forma más común de la enfermedad es la pulmonar.

La TB pulmonar tiene como principal síntoma la presencia de tos con expectoración mucoide o mucopurulenta por más de 15 días (2), denominándose a la persona que presente esta condición como sintomático respiratorio; esta tos puede estar acompañada por otros signos y síntomas como hemoptisis, fiebre, sudoración nocturna, malestar general, dolor torácico, astenia, anorexia y pérdida de peso.

La Organización Mundial de la Salud (OMS) para el año 2012 notificó 6.1 millones de casos incidentes a nivel mundial, de éstos 1.1 millones se presentaron en personas convivientes con el VIH de los cuales murieron 320000. Es una enfermedad con gran carga de morbilidad y mortalidad concentrada en los países en desarrollo (3).

En Colombia, de acuerdo con la información registrada por el Programa Nacional de Control de la Tuberculosis (PNCT), se tiene que el país pasó de una incidencia de 25.5 casos en el año 2002 a 23.97 casos por 100000 habitantes en 2013, es decir, que el comportamiento es constante en los últimos años, similar a lo que ocurre a nivel mundial donde se presenta un reducción lenta en el número de casos nuevos. Para el año 2013 se notificaron 12591 casos, 11294 nuevos y 1297 previamente tratados.

Con la reemergencia declarada por la OMS debida a la presencia de TB asociada a la coinfección TB/ VIH y la resistencia a fármacos, que producen las formas más graves de la enfermedad y complican el desarrollo de las actividades que realizan los PNCT, las cuales se han tenido que reorientar en busca de un diagnóstico oportuno y accesible, un tratamiento adecuado que se suministre en las condiciones de supervisión e incluso el abordaje institucional del tema de control de infecciones (4).
En el Departamento del Meta durante el año 2012 se reportaron 405 casos de tuberculosis todas las formas, para una tasa de incidencia de 44 casos por cada 100.000 Habitantes, el $60 \%$ de los casos ocurrieron en hombres y el $40 \%$ en mujeres; la tasa de incidencia en Villavicencio fue de 62,6 casos por cada 100.000 habitantes, aportando al departamento alrededor del $70 \%$ de los casos. Los abandonos al tratamiento en la cohorte 2012, tuvieron una tasa de 14,65\%; el aporte de Villavicencio fue del 65\% (5).

La adherencia a un tratamiento se define como la coincidencia entre la conducta del paciente y la orden del médico (6) y se presenta por una compleja interrelación de factores, entre los cuales se encuentran algunos derivados del paciente y su entorno económico, cultural y social y de otros derivados de la enfermedad en sí, del tratamiento farmacológico y sus características, así como aquellos derivados de los servicios de salud y del personal que atiende al paciente (7).

El abandono del tratamiento antituberculoso se define como la no asistencia a tomar tratamiento por más de 30 días consecutivos. Es una barrera importante para el control de la TB, por la relación con fracaso del tratamiento, desarrollo de farmacorresistencia, diseminación de la infección e incrementos en los costos de tratamiento. Entre las posibles causas de abandono se describen: la larga duración del tratamiento (6 a 12 meses), el alto número de pastillas, reacciones adversas a medicamentos antituberculosos (RAFAS) y desinformación sobre la enfermedad y su tratamiento (8).

Una de las principales causas de este problema han sido las elevadas tasas de abandono al tratamiento antes de los años noventa (50\%), y si bien la prevalencia de abandono en los últimos 5 años no supera el $5 \%$, este promedio no evidencia las altas tasas de varias ciudades y departamentos (9).

Debido a que la tuberculosis persiste en el escenario epidemiológico mundial, nacional y departamental a causa de la epidemia del virus de la inmunodeficiencia humana (VIH), la resistencia a múltiples medicamentos, las condiciones de pobreza de las poblaciones y los ineficientes sistemas de salud (10) se propuso, dentro de los Objetivos de Desarrollo del Milenio (ODM), detener y reducir la incidencia de la tuberculosis para el 2015 (11).
ISSN 1794-9831

E-ISSN 2322-7028

Vol. 12 No. 2

Jul - Dic 2015

Cúcuta, Colombia

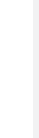


ISSN 1794-9831

E-ISSN 2322-7028

Vol. 12 No. 2

Jul - Dic 2015

Cúcuta, Colombia
La OMS trabaja en el cumplimiento de estos objetivos mediante la estrategia Alto a la Tuberculosis, en su primer componente que pretende proseguir la expansión de DOTS (Directly Observed Treatment Short/Tratamiento Acortado Estrictamente Supervisado) de calidad y mejorarlo. La estrategia DOTS ha demostrado ser costoefectiva para el control de la tuberculosis y ha sido implementada en casi todos los países del mundo (12).

"El abandono del tratamiento antituberculoso ha aumentado considerablemente en los últimos años, lo que a su vez ha aumentado la morbilidad y mortalidad por esta enfermedad, llevando al incremento en la incidencia y la prevalencia de casos de TB en el país" (13). El abandono del tratamiento antituberculoso se asocia a mayor contagio, resistencia a los antibióticos, aumento de costos y muerte (9); lo que se constituye un ingente desafío para los sistemas de salud y programas de prevención y control.

La TB se transmite por personas que tengan la enfermedad activa, a través de partículas emitidas con la tos, estornudo, al hablar o escupir, por el paciente bacilífero (con TB activa). Las gotas infecciosas (flügge's o droplets) son de un diámetro entre 0,5 a $5 \mu \mathrm{m}$, pudiéndose producir alrededor de 400.000 con un solo estornudo. Cada una de esas gotitas proveniente de un enfermo activo puede transmitir el microorganismo, especialmente sabiendo que la dosis infectante de la tuberculosis es considerablemente baja, de modo que la inhalación de una sola de las bacterias puede causar una infección (14).

La probabilidad de una transmisión eficaz aumenta con el número de partículas contaminadas expelidas por el enfermo, en lo bueno que sea la ventilación del área, la duración de la exposición y en la virulencia de la cepa del MT. Las personas con contactos frecuentes, prolongados, o intensos tienen un riesgo alrededor del $25 \%$ mayor de ser infectados. Para un fumador las posibilidades de enfermar se multiplican por 2,5. Un paciente con TB activa sin tratamiento puede infectar entre 10-15 personas por año. Otros riesgos incluyen aquellas áreas donde la TB es frecuente, en pacientes inmunodeprimidos con condiciones como malnutrición y VIH, poblaciones étnicas en alto riesgo y trabajadores de la salud sirviendo en regiones de alto riesgo (15).
La TB en el municipio de Villavicencio Meta, de acuerdo al perfil epidemiológico reportado en los últimos cinco años, es de los eventos de mayor incidencia; siendo la población joven y adulta quien con mayor frecuencia enferma. De acuerdo a la tabla de semaforización de los eventos de notificación obligatoria del departamento del Meta, 2007-2011 establecida en el Análisis de Situación en Salud (ASIS) del Departamento del Meta (16), la letalidad por tuberculosis, tuberculosis pulmonar y tuberculosis extra pulmonar tiene tasas similares en las dos primeras y superiores en la última, a las tasas nacionales de estos eventos; todas con tendencia al aumento.

\section{MATERIALES Y MÉTODOS}

Estudio descriptivo de corte transversal con enfoque cuantitativo. Fueron seleccionados 30 casos que corresponden al total de abandonos notificados entre los pacientes diagnosticados con TB pertenecientes a la cohorte 2012 y que formaron parte del programa de Tb departamental.

Se aplicó el instrumento diseñado por el investigador denominado Factores de Riesgo Asociados a la No Adherencia al Tratamiento Anti Tuberculosis sometido a revisión por expertos en la temática, quienes la aprobaron teniendo como base el instrumento diseñado por Salazar et al (17). La herramienta consta de variables sociodemográficas (10 ítems) y de caracterización de factores de riesgo para el abandono al tratamiento (11 items con opción de respuesta si o no, de rangos o escala).

Para la aplicación del instrumento se contactó a los pacientes con los datos obtenidos del registro de seguimiento a pacientes facilitado por PDCT a quienes se les solicitó consentimiento escrito para su utilización. El contacto inicialmente fue telefónico, las personas que no dieron respuesta a la primera llamada, se les realizó cinco intentos de localización, lográndose aplicar al 100\% de los abandonos. Una vez establecido el contacto, se concertó fecha y hora para la aplicación y previa firma del consentimiento informado por parte de los pacientes, se aplicó el cuestionario.

Los datos obtenidos fueron tabulados y analizados mediante la herramienta Microsoft Excel ${ }^{\circledR} 2007$ y Epi- 
info 7.0. La validez de los datos se obtuvo realizando listas desplegables para controlar las inconsistencias. Este trabajo no representa riesgo para las personas evaluadas según lo definido en la Resolución 08430 de 1993 del Ministerio de Protección Social, debido a que no se realiza ninguna intervención o modificación intencionada de las variables biológicas, fisiológicas, sicológicas o sociales de los individuos que participan en el estudio.

\section{OBJETIVOS}

\section{Objetivo general}

Determinar cuáles son los factores de riesgo asociados a la no adherencia al tratamiento anti tuberculoso en pacientes de la cohorte 2012 en el Municipio de Villavicencio Meta.

\section{Objetivos específicos}

- Identificar las características sociodemográficas de la población abordada.

Figura 1: Distribución por estado civil en los pacientes con TB.

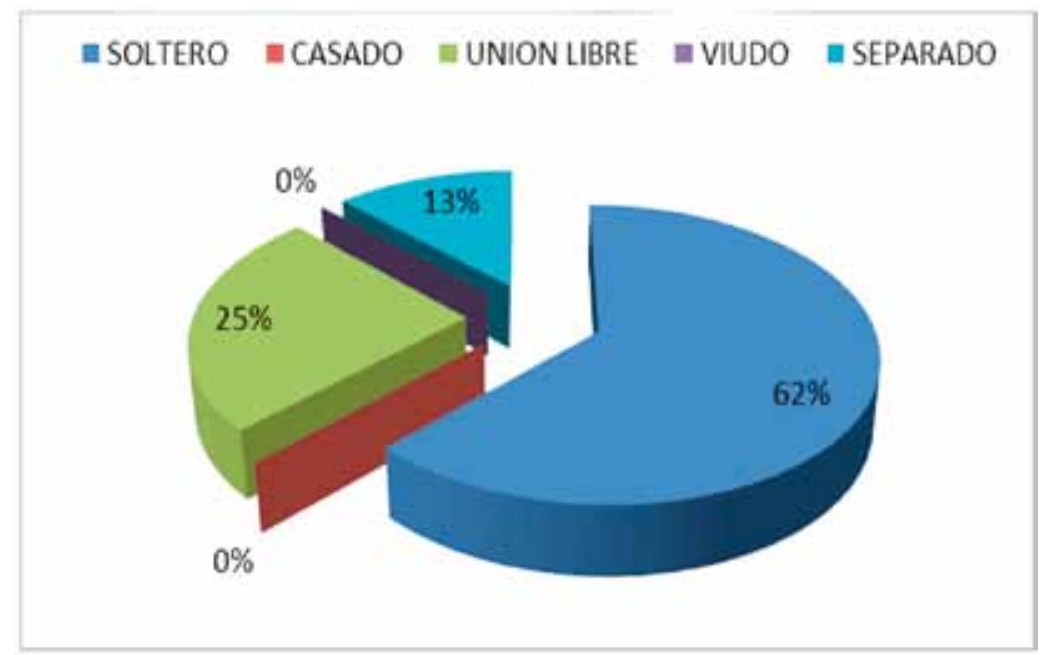

Fuente: Instrumento Factores de Riesgo Asociados a la No Adherencia al Tratamiento AntiTuberculosis, 2012.

Respecto a la vivienda, el $75 \%$ vive en vivienda familiar, el $12 \%$ en arriendo y el restante son habitantes de calle. El 100\% vive en la zona urbana en Villavicencio. La variable escolaridad mostró que el $37 \%$ no tiene estudio, el $25 \%$ tiene primaria incompleta, $25 \%$ primaria completa y el $12.5 \%$ tiene secundaria completa. El $62.5 \%$ está desempleado y
- Descubrir aspectos sobre estilos de vida, conocimientos y atención de la enfermedad de los pacientes.

- Describir las principales causas de abandono al tratamiento.

\section{RESULTADOS}

\section{Caracterización sociodemográfica}

El $75 \%$ de los pacientes pertenecen al género masculino; el promedio de edad es de 30 años con un mínimo de 21 y un máximo de 49 años. El 63\% tiene régimen subsidiado y pertenecen a Cajacopi (33\%), Comparta Capital Salud (17\% cada uno), y Caprecom $(16 \%)$.

La distribución por estado civil demuestra un alto porcentaje de solteros $(62 \%)$, seguido de unión libe y separados (figura 1). por lo tanto no tiene ningún ingreso aunque no tiene ninguna persona a cargo.

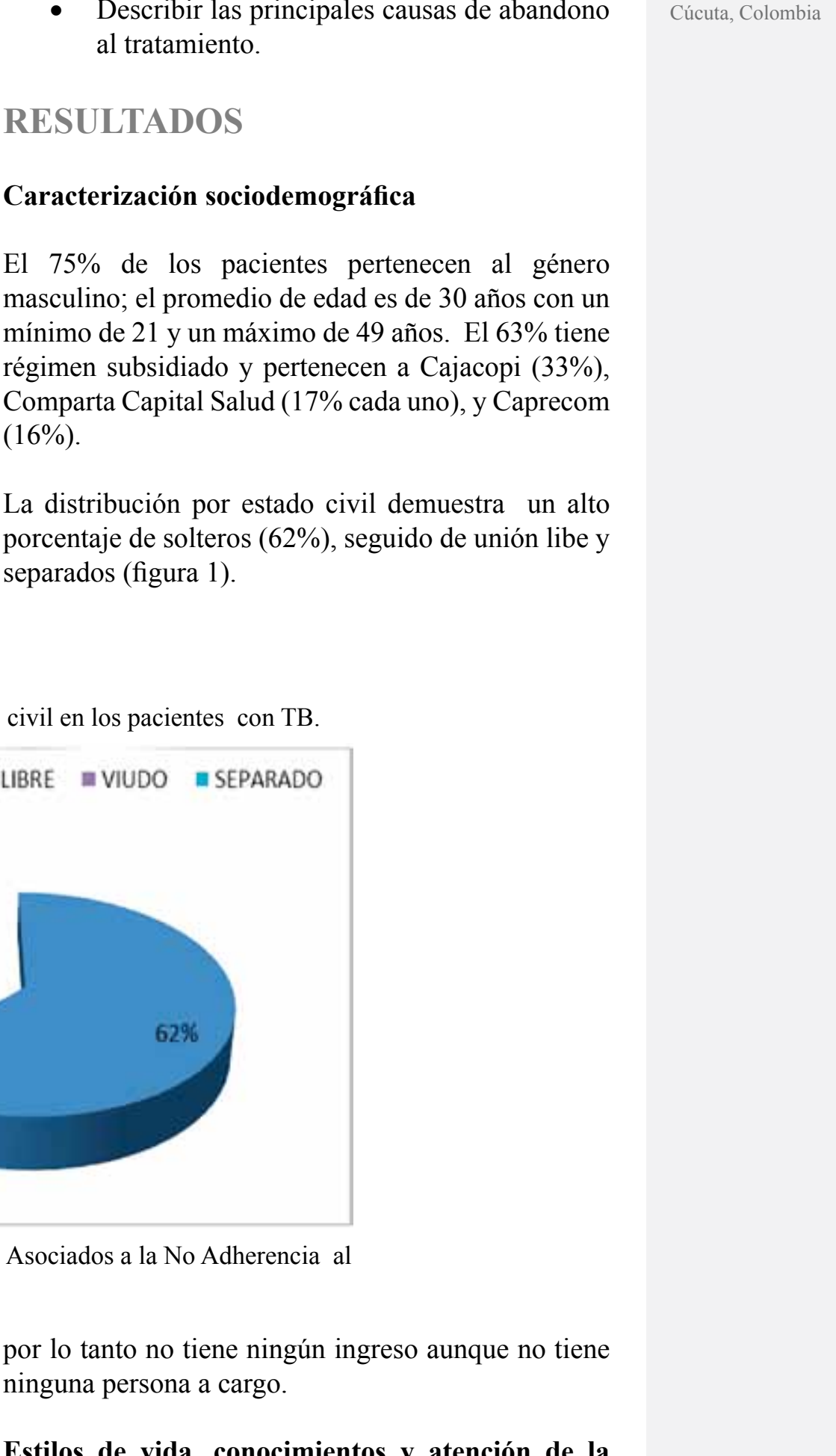

ISSN 1794-9831

E-ISSN 2322-7028

Vol. 12 No. 2

Jul - Dic 2015

Cúcuta, Colombia enfermedad

El $25 \%$ consume alcohol una vez al mes; el $25 \%$ 


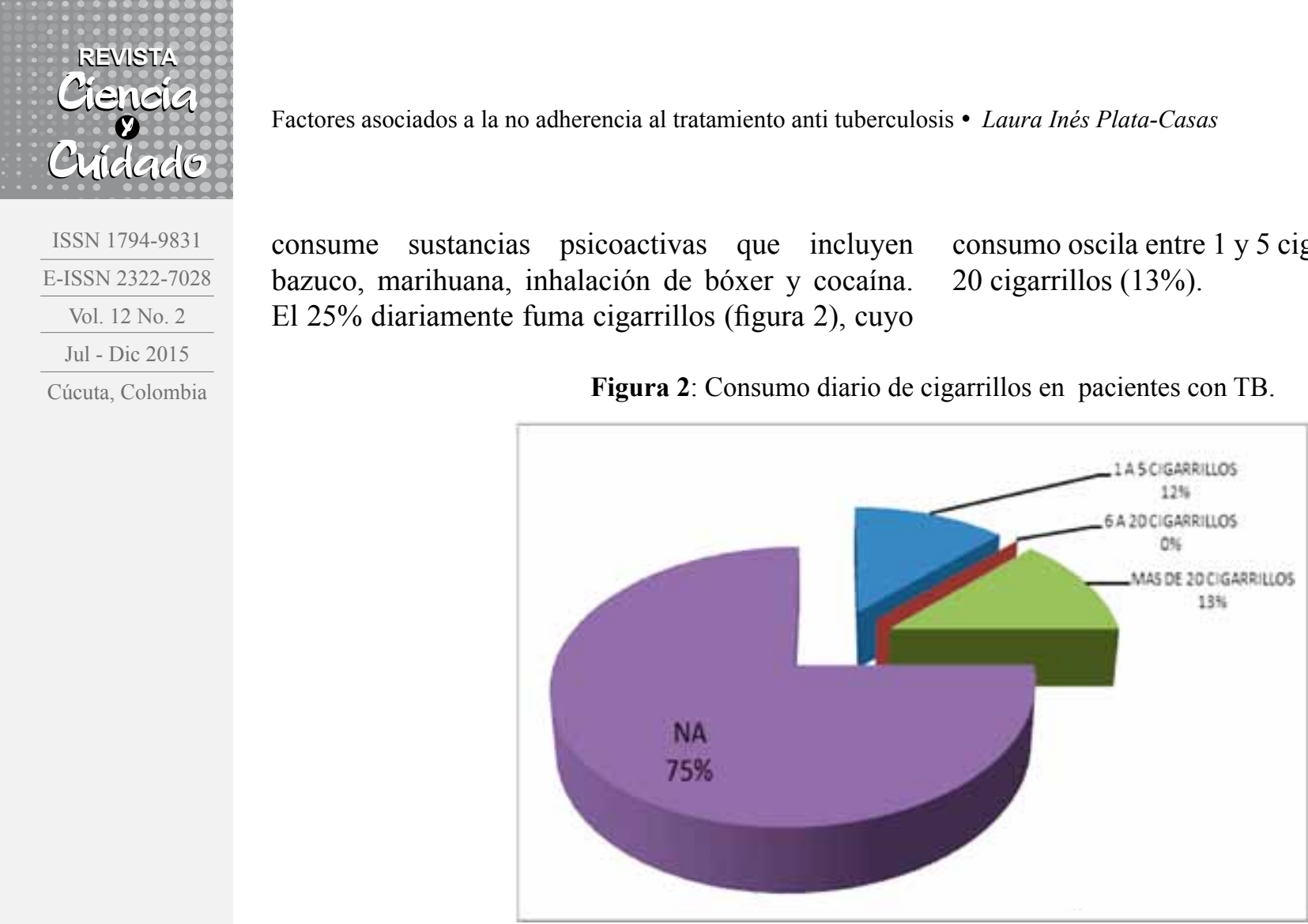

ISSN 1794-9831

E-ISSN 2322-7028

Vol. 12 No. 2

Jul - Dic 2015

Cúcuta, Colombi

Fuente: Instrumento Factores de Riesgo Asociados a la No Adherencia al Tratamiento Anti Tuberculosis, 2012.

El $62 \%$ conoce sobre tuberculosis; información obtenida principalmente a través del sector salud $(50 \%)$, sin embargo el conocimiento sobre medicamentos que consumen es bajo.

\section{Causas de abandono}

El $80 \%$ presenta RAFAS especialmente vértigo, nauseas, vómito, dispepsia y artralgias. Respecto a las principales causas de abandono están los efectos adversos a los medicamentos (58\%), inadecuada atención del personal de salud (43\%), dificultad de acceso al servicio (32\%); cambio de domicilio, fallas en la información dada por parte del personal de salud, cambio de empresa promotora de salud (EPS) (10\% cada una).

Los efectos adversos (figura 3) más comunes que se presentaron fueron: vértigo $(80 \%)$, nauseas $(52 \%)$, vómito, dispepsia y artralgias con $31 \%$ cada uno.

Figura 3: Reacciones adversas a los medicamentos.

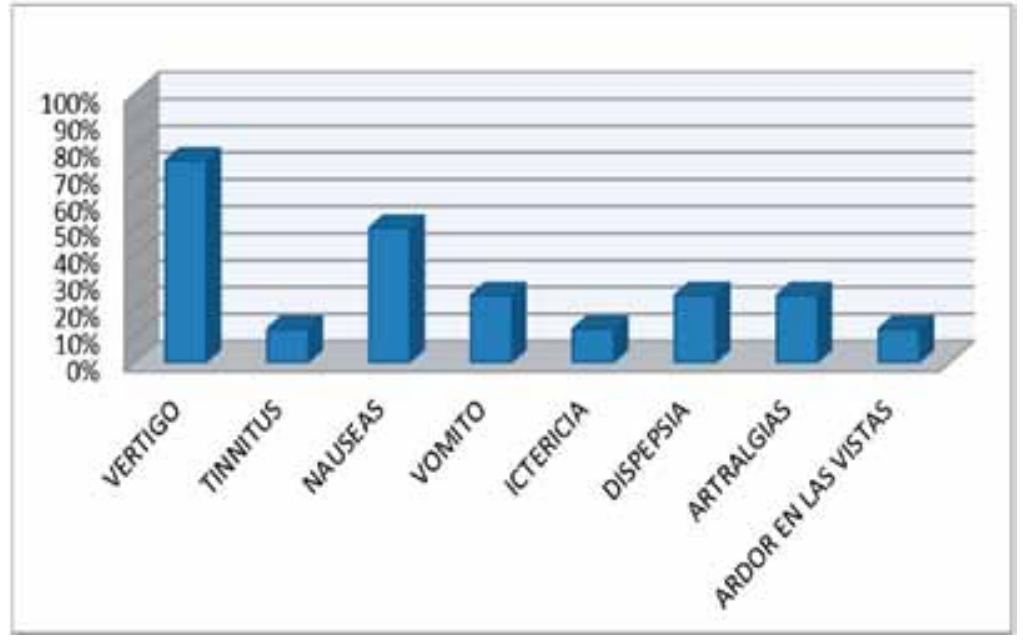

Fuente: Instrumento Factores de Riesgo Asociados a la No Adherencia al Tratamiento Anti Tuberculosis, 2012. 
El $62 \%$ consideran que el trato por el personal de salud fue bueno, la atención fue inmediata una vez llegó al programa; sin embargo, el tiempo que usan para el traslado de la casa a la EPS es de menos de una hora en el 50\% de los casos; entre una y dos hora en el $37 \%$ de los casos y el restante entre tres y cinco horas.
Después de que les fue comunicado el diagnostico, los pacientes manifiestan sentir tristeza (98\%), angustia (82\%), soledad (81\%) y depresión (74\%). Otras manifestaciones en menor proporción fueron: sentimientos de inferioridad (39\%), invalidez (38\%) y abandono (22\%), tal y como se muestra en la figura 4.
ISSN 1794-9831

E-ISSN 2322-7028

Vol. 12 No. 2

Jul - Dic 2015

Cúcuta, Colombia

Figura 4: Sentimientos post diagnóstico.

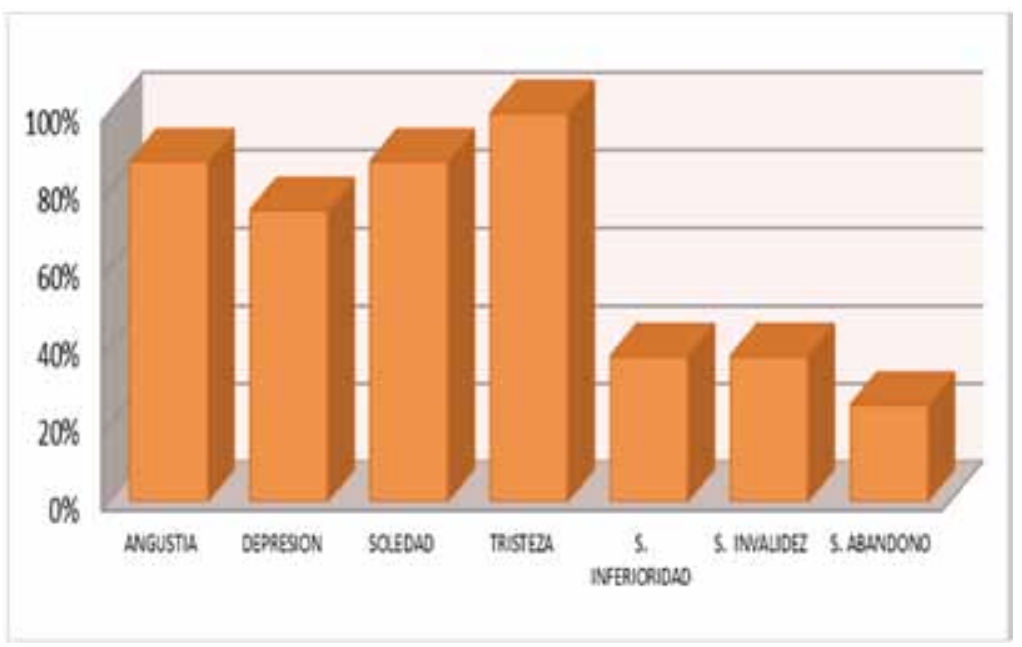

Fuente: Instrumento Factores de Riesgo Asociados a la No Adherencia al Tratamiento Anti Tuberculosis, 2012.

El 60\% una vez son diagnosticados con TB limitan sus actividades sociales, en la misma proporción olvida las cosas que le suceden recientemente, el 50\% tiene dificultades para razonar o resolver problemas y solo un $38 \%$ recibe visitas. El $62 \%$ manifiesta sentirse mucho mejor después del tratamiento.
Con relación a los factores sociales se encuentra hacinamiento en el $67 \%$ de los casos y el $62 \%$ están desempleados.

Las causas de abandono (figura 5) contemplaron principalmente los efectos adversos (58\%), la atención del profesional de salud (46\%) y la dificultad de acceso al servicio (32\%).

Figura 5: Causas de abandono del tratamiento.

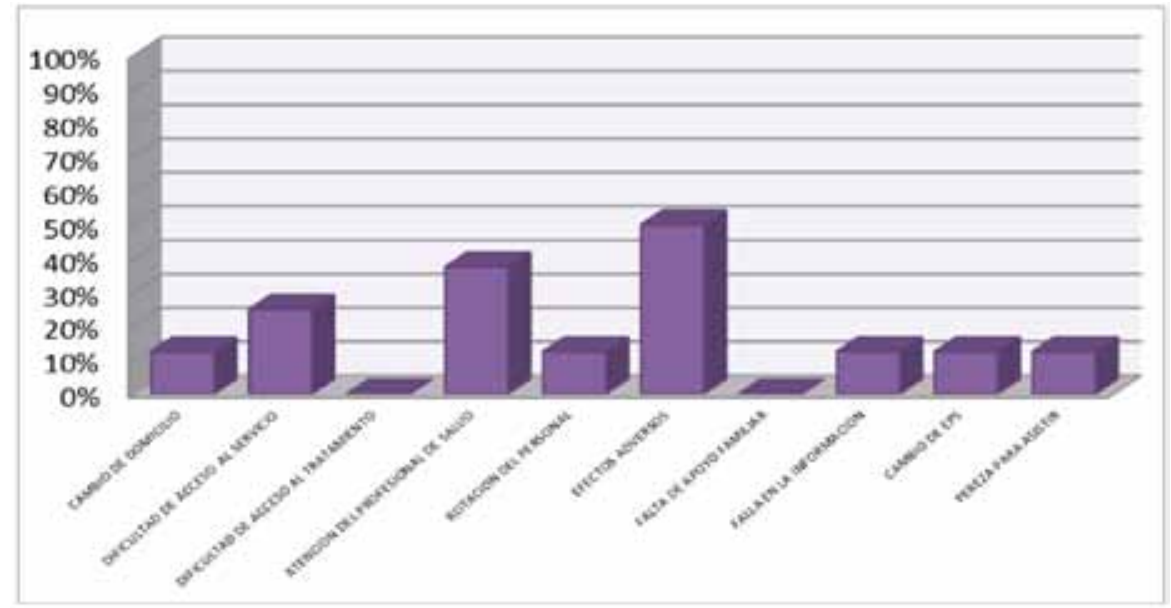

Fuente: Instrumento Factores de Riesgo Asociados a la No Adherencia al Tratamiento AntiTuberculosis, 2012. 
ISSN 1794-9831

E-ISSN 2322-7028

Vol. 12 No. 2

Jul - Dic 2015

Cúcuta, Colombia

\section{DISCUSIÓN}

\section{Caracterización sociodemográfica}

Los resultados indican respecto a la caracterización por género, que hay concordancia con lo mencionado por la OMS y la literatura, pues es bien conocido el hecho de que los índices de notificación de tuberculosis sean más elevados en los hombres, lo cual puede obedecer en parte a diferencias epidemiológicas (en cuanto a exposición, riesgo de infección y progresión desde el estadio de infección al de enfermedad). Igualmente se ha establecido en estudios al respecto, que es un factor de riesgo de abandono de la farmacoterapia antituberculosa, el sexo masculino (18-24).

La TB es la patología reemergente más frecuente en las personas jóvenes y tiende a ser más agresiva en el grupo que va desde los 15 hasta los 45 años. Es relevante el hecho que el $63 \%$ de los pacientes pertenecen al régimen de aseguramiento en salud subsidiado, lo que puede indicar que el desarrollo de TB está ligado a la escasez de recursos socioeconómicos (25-26). Se ha reportado que el estado civil casado y la convivencia familiar ofrecen al paciente un mecanismo de adherencia importante al tratamiento (27); en este caso, un alto porcentaje no tiene una pareja, lo que puede influir en la decisión de abandono de tratamiento.

Estudios han evidenciado la asociación entre las condiciones de la vivienda y el abandono del tratamiento, pues la falta de vivienda o lo inconveniente de la misma, tanto en espacio como en la falta de servicios básicos, provoca hacinamiento e insalubridad en los ocupantes (28); en este trabajo, la mayoría de los encuestados habitaban en viviendas familiares con condiciones sanitarias marcadamente inadecuadas. La enfermedad se desarrolla allí donde existe la precariedad social, la desnutrición y la carencia de recursos (29-30).

El nivel educativo afecta la no adherencia al tratamiento, pues a menor nivel, mayor probabilidad de abandono (31-32); los resultados de este estudio, revelaron concordancia al respecto. El bajo nivel educativo limita la comprensión de la enfermedad, sus implicaciones en cuanto a contagio, importancia de adherencia al tratamiento, acentuamiento de conceptos culturales falsos que inciden sobre la capacidad de comprensión de la información suministrada por el personal de salud.

De igual forma, la falta de empleo es un factor de riesgo importante; en este aspecto cabe señalar que más de la mitad está desempleado, lo que evidencia, nuevamente, que la falta de ingresos o el tener un empleo esporádico o eventual es un factor de riesgo importante (33).

\section{Estilos de vida, conocimientos y atención de la enfermedad}

El consumo de sustancias psicoactivas, así como el hábito de fumar, tal y como lo demuestran referentes anteriores (34-36) es un factor de riesgo; coincidiendo con los resultados del estudio. Respecto al conocimiento de la enfermedad, los participantes conocen sobre ella, información que es ofrecida especialmente por los servicios de salud; sin embargo la falta de información respecto a los medicamentos y sus afectos adversos, unidos al bajo nivel educativo, pueden estimular la falta de adherencia.

La inadecuada atención del personal de salud, los cambios en el domicilio por parte del paciente, movilidad entre aseguradores, motivaron la decisión de abandonar el tratamiento. En este estudio, la deserción no estuvo relacionada con residencia en el medio rural; contrario a lo que se demostró en un estudio en México (37) donde hubo una asociación fuerte entre abandono y residencia en el medio rural. Sin embargo, es relevante el hecho que deban invertir un elevado número de horas -esperando- para poder acceder a los servicios de salud, lo cual afecta la adherencia al tratamiento.

\section{Causas de abandono}

Las causas que prevalecen en el abandono del tratamiento fueron los efectos adversos a los medicamentos, especialmente de orden digestivo, así como la atención de los profesionales de la salud, la mejoría sintomática y las dificultades de acceso al servicio.

En un estudio realizado en España (38), se señala que existe una relación directa entre la dosificación, la duración y los efectos secundarios de los tratamientos, con la conducta de cumplimiento. En él se plantea 
entre otros que la tasa de adherencia disminuyó en la medida que la terapia se alargó y provocaba mayor cantidad de efectos secundarios indeseados, lo cual coincide con el presente estudio, donde los efectos adversos a los medicamentos, fueron causa de abandono.

Se ha reportado en otras investigaciones (39-44) que el desagrado con la atención recibida por los profesionales en salud, es tomada como un obstáculo institucional que ocasiona el abandono al tratamiento; concordando con los resultados encontrados.

El abandono por mejoría sintomática se puede llegar a interpretar como parte de una conciencia inapropiada de la enfermedad, al respecto Flores y Rivas (45), encontraron que el sentir mejoría constituía el $44,4 \%$ de las causas de abandono del tratamiento farmacológico antihipertensivo en pacientes.

Es necesario vigilar estrictamente la evolución de los pacientes, sensibilizarlos sobre estas manifestaciones, capacitar al personal de salud para que la educación en esos aspectos se enfoque especialmente hacia ellos, e involucrar programas psicosociales de apoyo.

Los sentimientos de tristeza, soledad, angustia y depresión, así como la sensación de inferioridad, invalidez y abandono fueron relevantes, afectando de forma determinante su vida diaria, teniendo que replantear prioridades, adaptarse a nuevos estilos de vida, convivir lo mejor posible con la enfermedad, limitando las actividades que realizan, la interacción social y el desenvolvimiento en la resolución de problemas cotidianos; haciendo difícil el proceso de adaptación a la vida diaria en la mayoría de los casos, si no encuentran el apoyo psicológico y soporte social suficiente.

\section{CONCLUSIONES}

Los factores de riesgo hallados asociados a la no adherencia al tratamiento, tienen que ver con el paciente, los servicios de salud y el tratamiento; se encontraron factores no modificables como la edad $y$ el género, $y$ factores modificables como estilos de vida inadecuados.

Los efectos secundarios de los medicamentos (80\%), el no manejo profiláctico de ellos y la inadecuada relación entre el paciente y personal de salud (43\%), contribuyen a la falta de adherencia al tratamiento.

Factores modificables como estilos de vida inadecuados, consumo de alcohol (25\%), sustancias psicoactivas $(25 \%)$ y el fumar $(25 \%)$ son aspectos importantes a tener en cuenta en pacientes con TB, al favorecer la no adherencia al tratamiento.

El desconocimiento sobre la enfermedad (38\%) la baja información brindada (50\%) especialmente sobre medicamentos y RAFAS, son factores trascendentes para abordar en los pacientes diagnosticados con tuberculosis, pues favorecen que el paciente no dé continuidad y termine su tratamiento.

Los resultados muestran que el adulto joven (rango entre 31 y 49 años) de sexo masculino (75\%), afiliado a régimen subsidiado (63\%), de estado civil soltero $(62 \%)$ y con bajo nivel educativo $(87 \%)$ es más proclive a abandonar el tratamiento.

Las variables psicológicas y sociales se convierten en predictores de probables abandonos o irregularidades en el tratamiento antituberculoso. La falta de atención psicológica y social sobre los síntomas depresivos que acompañan a la enfermedad física, así como la poca actividad soportiva de la red social, fijan el abandono del tratamiento.

Los factores de riesgo de no adherencia al tratamiento para tuberculosis hallados como son: RAFAS, factores no modificables como la edad y el género, y factores modificables como estilos de vida inadecuados, los sentimientos de tristeza, soledad, angustia y depresión, así como la sensación de inferioridad, coinciden con los factores revelados en otros estudios nacionales e internacionales realizados.

La articulación intersectorial de los programas de control de la tuberculosis con otras estancias como la economía, la política, y demás que velan por el bienestar de las poblaciones; es fundamental y urgente, para realizar acciones en frente común de prevención de no adherencia al tratamiento.

Es importante introducir modificaciones en los servicios de salud, especialmente para evitar el desagrado con la atención recibida por los profesionales en salud en el manejo programático del paciente con TB. 
ISSN 1794-9831 E-ISSN 2322-7028

Vol. 12 No. 2

Jul - Dic 2015

Cúcuta, Colombia

Es necesario incorporar en la tarjeta de tratamiento de los pacientes, variables que permitan detectar de forma rápida a los pacientes con un posible riesgo de abandono de tratamiento.

\section{CONFLICTO DE INTERESES}

El autor declara no tener ningún conflicto de intereses. 


\section{REFERENCIAS BIBLIOGRÁFICAS}

1. World Health Organization. Global Tuberculosis Report 2012. [Internet]. Geneve: Switzerland: WHO; 2012 [consultado marzo de 2015]. Disponible en: http://apps.who.int/iris/bitstream/10665/75938/1/9789241564502_eng. pdf

2. Ministerio de Salud. Protocolo de vigilancia en salud pública Tuberculosis. Bogotá DC: Ministerio de Salud; 2015.

3. World Health Organization. Global tuberculosis control: epidemiology, strategy, financing report. [Internet]. Ginebra: WHO; 2011 [consultado abril de 2015]. Disponible en: http://apps.who.int/iris/ bitstream/10665/44728/1/9789241564380_eng.pdf

4. Instituto Nacional de Salud. Informe: informe del evento tuberculosis, hasta el periodo epidemiológico 13 del año 2012 - cierre. [Internet]. Bogotá: Instituto Nacional de Salud; 2012 [consultado mayo de 2015]. Disponible en http://www.ins.gov.co/lineas-de-accion/Subdireccion-Vigilancia/Informe\%20de\%20Evento\%20Epidemiolgico/ TUBERCULOSIS\%202012.pdf

5. Gobernación del Meta. Informe: Análisis Epidemiológico de Tuberculosis 2012 y 2013. Villavicencio Meta. [Internet]. Villavicencio: Gobernación del Meta; 2012 [consultado mayo de 2015]. Disponible en: http://www.meta. gov.co/es/analisis-epidemiologico-de-tuberculosis-en-el-departamento-del-meta/

6. Basterra GM. El cumplimiento terapéutico. Pharm Care 1990 (1): 97-106.

7. Garner P. GAT maker DOT work? Lancet 1998; 352:1326-7.

8. Ministerio de la Protección Social. Programa de Apoyo a la Reforma de Salud Universidad Nacional de Colombia. Instituto de Investigaciones Públicas. Guías de promoción de la salud y prevención de enfermedades en la salud pública. Tomo II. [Internet]. Bogotá D.C.: Programa de Apoyo a la Reforma de Salud - PARS; 2007 [consultado mayo de 2015]. Disponible en: https://www.minsalud.gov.co/Documentos\%20y\%20Publicaciones/GUIAS\%20 DE\%20ATENCION\%20-TOMO\%20DOS.pdf

9. Culqui DR, Munayco CV, Grijalva CG, Cayla JA, Horna-Campos 'O, Alva K, et al. Factores asociados al abandono de tratamiento antituberculoso convencional en Perú. Revista Archivos de Bronconeumologia. 2012; 48 (5):150-5.

10. World Health Organization. Tuberculosis facts Sheet No. 104. [Internet]. Geneva: World Health Organization; 2015 [consultado marzo de 2015]. Disponible en: http://www.who.int/mediacentre/factsheets/fs 104/en/

11. Los objetivos de desarrollo del milenio. Informe 2010. [Internet]. New York: ONU; 2010 [consultado 31 de agosto de 2014]. Disponible en: http://www.un.org/es/millenniumgoals/pdf/MDG_Report_2010_SP.pdf

12. World Health Organization. The stop TB strategy. Building on and enhanching DOTS to meet the Tb-related millennium development goals. [Internet]. Geneva: WHO; 2006 [consultado 15 de abril de 2011]. Disponible en: http://www.who.int/tb/publications/2006/stop_tb_strategy.pdf

13. Navarro-Quintero C, Rueda-Rincón JL, Mendoza-Ojeda JL. Factores asociados al abandono del tratamiento antituberculoso en pacientes con TB. Rev. cienc. cuidad. 2013; 10(1): 19-27.

14. Cole E, Cook C. Characterization of infectious aerosols in health care facilities: an aid to effective engineering controls and preventive strategies. Am J Infect Control [Internet]. 1998 [consultado abril de 2015]; 26 (4): 453-64. DOI:10.1016/S0196-6553(98)70046-X. PMID 9721404

15. Raviglione MC, O’Brien RJ. Tuberculosis. En: Kasper DL, Braunwald E, Fauci AS, Hauser SL, Longo DL, Jameson JL, Isselbacher KJ, eds. Harrison's Principles of Internal Medicine. 16a ed. EEUU: McGraw-Hill Professional. 2004. p. 953-66. [consultado abril de 2015]. DOI: 10.1036/0071402357. ISBN 0071402357.

16. Departamento del Meta. Informe: Análisis de Situación en Salud con el Modelo de los Determinantes Sociales de Salud. [Internet]. Villavicencio: Secretaria de Salud del Meta; 2014 [consultado abril de 2015]. Disponible en: http://saludmeta.gov.co/apc-aa-files/373537626531346333337613535333031/asis_meta_07_07_2014.pdf

17. Salazar CL, Uribe MP, Zuloaga WA, Ríos JF, Montes F. Factores de riesgo para el abandono del tratamiento contra la tuberculosis en la ciudad de Medellín. (Enero 2000-Junio2001). Revista Ces Medicina. Medellín. Ene- Jul. 2004; 18(1): 28-29.

18. Tuberculosis: la enfermedad reemergente con mayor incidencia entre los jóvenes. $9^{\circ}$ Congreso Internacional de Enfermedades Infecciosas, Informe Especial: Cobertura Periodística. Buenos Aires, Abril del 2000.

19. Soza-Pineda NI, Pereira SM, Barreto ML. Abandono del tratamiento de la tuberculosis en Nicaragua: resultados de un estudio comparativo. Rev Panam Salud Publica. 2005; 17(4):271-8.

20. Oliveira HB, Moreira FC. Abandono de tratamento e recidiva da tuberculose: aspectos de episódios prévios, Campinas SP, Brasil, 1993- 1994. Rev Saude Publica. 2000;34(5):437-43.

21. Manjarrez EM, Serrano MV, Cano PG, Verduzco GE, Escandón RC, Escobedo PJ. Principales causas de abandono de tratamiento contra la tuberculosis pulmonar. Gac Med Mex. 1993;129(1):57-62.

22. Lima MB, Mello DA, Morais APP, Silva WC. Estudo de casos sobre abandono do tratamento da tuberculose: 
avaliação do atendimento, percepção e conhecimentos sobre a doença na perspectiva dos clientes, Fortaleza, Ceará, Brasil, 1996. Cad Saude Publica. 2001; 17(4):877-85.

23. Deheinzelin D, Takagaki TY, Sartori AMC, Leite OHM, Neto VA, Carvalho CRR. Fatores preditivos de abandono de tratamento por pacientes com tuberculose. Rev Hosp Clin Fac Med Sao Paulo. 1996;51(4):131-135.

24. Burman WJ, Cohn DL, Rietmeijer CA, Judson FN, Sbarbaro JA, Reves RR. Short-term incarceration for the management of noncompliance with tuberculosis treatment. Chest. 1997;112(1):57-62.

25. Ribeiro SA, Amado VM, Camelier AA, Fernandes MMA, Schenkman S. Estudo de casocontrole de indicadores de abandono em doentes com tuberculose. J Pneumologia. 2000;26(6):291-6.

26. Bothamley GH. Tuberculosis and social exclusion: new approach is needed. BMJ. [Internet]. 2006 [consultado marzo de 2015]; 333(7560):200. Disponible en: http://www.ncbi.nlm.nih.gov/pmc/articles/PMC1513503/

27. Lönnroth K, Jaramillo B, Williams BG, Dye C. Raviglione M. Drivers of tuberculosis epidemics: The role of risk factors and social determinants. Social Science \& Medicine [Internet]. June 2009 [consultado marzo de 2015]; 68(12):2240-6. Disponible en: http://www.sciencedirect.com/science/article/pii/S0277953609002111

28. Sumartojo E. When tuberculosis treatment fails. A social behavior account of patient adherence. Am Rev Respir Dis 1993; 147:1311-20.

29. Mangtani P, Jolley DJ, Watson JM, Rodrigues LC. Socioeconomic deprivation and notification rates for tuberculosis in London during 1892-1991. Br Med J 1995; 310:963-6.

30. Hadad-Hadad JL, Valdés-Llanes E. La protección social en salud como enfrentamiento a una crisis económica. Rev Cubana Salud Pública [Internet]. 2010 [consultado 5 de enero de 2010]; 36 (3): 235-248. Disponible en: http:// scielo.sld.cu/scielo.php?pid=S0864-34662010000300008\&script=sci_arttext

31. Fernández-Fernández M, Lara-Alfredo J, Rodríguez-Vargas L, Carreras-Corzo L, Castañeda-Hilda G. Tuberculosis, comportamiento de la mortalidad en pacientes de 60 años de edad o más. Rev Cubana Med Gen Integr [Internet]. 2012 [consultado 7 marzo de 2010]; 28 (2): 55-64. Disponible en: http://scielo.sld.cu/scielo.php?script=sci_ arttext\&pid=S0864-21252012000200006\&lng=es.

32. Galván F, Santiuste C. Factores relacionados con el cumplimiento de la quimioprofilaxis contra la tuberculosis. Med Clín (Barc) 1998; 11:655-7.

33. Ferrer X, Kirschbaum A, Toro J, Jadue J, Muñoz M, Espinoza A. Adherencia al tratamiento de la tuberculosis del adulto en Santiago, Chile. Bol Oficina Sanit Panam 1991; 111:423-31

34. Burman WJ, Cohn DL, Rietmeiyer CA, Judson FN, Sbarbaro JA, Reves RR. Noncompliance with directly observed therapy for tuberculosis. Chest. 1997; 111(5):1168-73.

35. Jiménez GD, López PD, Medrano MJ, Valle MJ. Factores asociados al abandono de pacientes bacilíferos al programa de control de tuberculosis, Managua, Nicaragua, 1998- 1999. Managua: Centro de Investigaciones y Estudios de la Salud (CIES-UNAN); 1992; 1-42.

36. Albuquerque MFM, Leitão CCS, Campelo ARL, Souza WV, Salustiano A. Fatores prognósticos para o desfecho do tratamento da tuberculose pulmonar em Recife, Pernambuco, Brasil, 1994-1999. Rev Pan Salud Pública 2001;9(6):368-74.

37. González-Tapia M, Vivas-Bombino L. Tuberculosis pulmonar y tabaquismo en la atención primaria en salud. Rev Ciencias Médicas [Internet]. Sept-oct 2012; 16(5): 35-43.

38. Colectivo de autores. Cumplimiento e incumplimiento terapéutico en el tratamiento antibiótico. Madrid: Ediciones Doyma; 1997.

39. García LF, Jaramillo E. La tuberculosis: Un reto que debemos enfrentar. Rev Biomédica Instituto Nacional de Salud. Bogotá, Colombia. 2004; 24 (Supl.1):5-9.

40. Homedes N, Ugalde A. ¿Qué sabemos del cumplimiento de los tratamientos médicos en el tercer mundo? Bolivia. Ofic Sanit Panam 1994; 116: 491-517.

41. Albuquerque MF. Pessoa Militão de; Ximenes R, Arraes de Alencar; LucenaSilva N. et al. Factors associated with treatment failure, dropout, and death in a cohort of tuberculosis patients in Recife, Pernambuco State, Brazil. Cad. Saú de Pública. 2007; 23 (7): 1573-1582.

42. Center for Diseases Control. Essential components of a tuberculosis program. Morb Mort Wkly Rep. 1994; 44:1-16.

43. World Health Organization. What is DOTS? A Guide to Understanding the WHO-recommended TB Control Strategy known as DOTS.WHO/CDS/ CPC/TB/99.270; 1999.

44. Manjarrez EM, Serrano MV, Cano PG, Verduzco GE, Escandón RC, Escobedo PJ. Principales causas de abandono de tratamiento contra la tuberculosis pulmonar. Gac Med Mex 1993; 129(1): 57-62.

45. Flores TR, Rivas VJ. Prevalencia de diabetes mellitus, obesidad, hipertensión arterial, hipercolesterolemia, como factores de riesgo de enfermedad cardiovascular y cerebrovascular en la población adulta del distrito de Chiclayo. Febrero-Abril 1995. [Tesis] Perú: Universidad Nacional Pedro Ruiz Gallo Lambayeque; 1995. 